\title{
Inverse Semihypergroups
}

\author{
NABILAH HANI ABUGHAZALAH \\ Department of Mathematical Sciences \\ Princess Nourah bint Abdulrahman University \\ P.O.Box 84428, Riyadh 11671 \\ SAUDI ARABIA
}

\begin{abstract}
The concept of inverse semihypergroup is a generalization of the concept of inverse semigroup. In this paper we defined an orthodox semihypergroup $S$ as it is a regular semihypergroup in which the set of idempotents of $S$ forms a subsemihypergroup. We also presented a new definition of inverse semihypergroup $S$ as each element $x \in S$ has a unique inverse $s^{-1}$ in $S$, and we provided many results related to inverse semihypergroups, regular semihypergroups and orthodox semihypergroups.
\end{abstract}

Key-Words: Semihypergroup, Inverse semihypergroup, Regular semihypergroup, Orthodox semihypergroup

Received: March 25, 2020. Revised: October 7, 2020. Accepted: October 20, 2020. Published: October 31, 2020.

\section{Introduction}

From several points of view, the theory of hyperstructures has been researched and extended to many fields of mathematics, computer science and logic. In a classical algebraic structure the composition of two components yields an element whereas in hyperstructures composition of two components could be a non-empty set. From there on numerous mathematicians started to work in this field and composed numerous enquire about articles and books on diverse hyperstructures.

A French mathematician F. Marty [1] proposed the theory of hyperstructures at the 8th Congress of Scandinavian Mathematicians in 1934. He gave out a few papers on hypergroups, using them in various settings: algebraic structures, rational fractions, and noncommutative groups. A fair generalization of classical algebraic structures is the algebraic hyperstructure.
In 1953, the Romanian algebraist Mihail Benado introduced the concept of hyperlattice in the paper "Asupra unei generălizari a noțiunii de structură" [2]. Benado provides two identical concepts of hyperlattice in this work and some examples as well.

A hyperring is a ring generalization where a hyperoperation is one of the operations. Similarly, a hyperfield is a hyperstructure that, in the above sense, generalizes the normal field definition. Marc Krasner introduced the definition of hyperfield in [3], [4] in relation to his studies in valued fields.

The concept of a semihypergroup is a generalization of a semigroup. As of late Davvaz has composed a book [5] on semihypergroups. Recent book on hyperstructures [6] points out to their applications in cryptography, codes, automata, probability, geometry, lattices, binary relations, graphs, and hypergraphs.

The general aspects of the theory, the re- 
lations with groups, and complex applications of geometry were researched during the 1940s. Significant improvement has been made since the 1970s, when the work field grew. Numerous authors considered particular points of semihypergroups, for instance, Corsini [7], Davvaz and Poursalavati [8]. Simplifable semihypergroups was investigated by Guţan [9] and Leoreanu [10]. Some results on congruences on semihypergroups were presented by Davvaz [11]. Bonansinga and Corsini [12] presented polygroups by Comer which called Quazi- canonical hypergroups. Fasino and Freni [13] generalized the definition of hypergroups of type $U$ to semihypergroups of type $U$. Hasankhani [14] presented the definition of an ideal in a left(right) semihypergroup and the link between ideals and greens relations were discussed. Adequate conditions for a locally compact semihypergroups are created by Onipchuk [15].

An inverse semipolygroup was introduced by Sutasinee Wannusit and Poonchayar Patthanangkoor [16]. They defined it as: Let $(S, \star)$ be a semipolygroup. It call an inverse semipolygroup if there exists a unique unary operation $x \rightarrow x^{-1}$ on $S$ with the properties

$$
\begin{gathered}
\left(x^{-1}\right)^{-1}=x, x \in x \star x^{-1} \star x, \\
x \in y \star z \text { implies } y \in x \star z^{-1} \text { and } \\
z \in y^{-1} \star x \forall x, y, z \in S .
\end{gathered}
$$

And some properties were presented.

In this paper we define an inverse semihypergroup by generalizing the definition of an inverse semigroup as it is defined as: Let $(S,$.$) be a semigroup. We say that x^{-1}$ is the inverse of $x$ if and only if

$$
x x^{-1} x=x \text { and } x^{-1}=x^{-1} x x^{-1} .
$$

If for every element $x \in S$ there is a unique inverse of $x$, then we call $(S,$.$) an inverse$ semigroup.
In this paper, we try, based on the paper of Lawson on inverse semigroups [17], to define inverse semihypergroups and prove some basic properties of inverse semihypergroups in analogy with those of inverse semigroups. We include several examples and counter-examples that demonstrate the complexity of the theory we are creating. We'll see that inverse semigroups and inverse semihypergroups are equivalent in many respects, but they are very different in many other respects.

\section{Basic Definition}

Definition 2.1. Let $S$ be a non-empty set and let $\star: S \times S \rightarrow P(S)^{*}$ be a mapping defined by $\star(x, y)=x \star y$. We call $(S, \star) a$ hypergroupoid. If $T_{1}, T_{2}$ are two non-empty subsets of $S$ and $x \in S$ then

$$
T_{1} \star T_{2}=\bigcup_{\substack{t_{1} \in T_{1} \\ t_{2} \in T_{2}}} t_{1} \star t_{2},
$$

$T_{1} \star x=T_{1} \star\{x\}, \quad x \star T_{2}=\{x\} \star T_{2}$

Definition 2.2. We call $(S, \star)$ a semihypergroup if

$$
(x \star y) \star z=x \star(y \star z) \forall x, y, z \in S,
$$

which implies that

$$
\bigcup_{u \in x \star y} u \star z=\bigcup_{v \in y \star z} x \star v .
$$

Definition 2.3. Let $(S, \star)$ be a semihypergroup and $A$ be a non-empty subset of $S$. We call $A$ a subsemihypergroup if $\forall x, y \in A$ : $x \star y \subseteq A$.

Example 2.4. Let $S=\{a, b, c, d\}$ and let the operation $\star$ defined by the following hyperoperation table: 


\begin{tabular}{c|c|c|c|c}
$\star$ & $\mathrm{a}$ & $\mathrm{b}$ & $\mathrm{c}$ & $\mathrm{d}$ \\
\hline $\mathrm{a}$ & $\{\mathrm{a}, \mathrm{c}, \mathrm{d}\}$ & $\mathrm{d}$ & $\{\mathrm{a}, \mathrm{c}, \mathrm{d}\}$ & $\mathrm{d}$ \\
\hline $\mathrm{b}$ & $\{\mathrm{a}, \mathrm{c}, \mathrm{d}\}$ & $\mathrm{d}$ & $\mathrm{c}$ & $\mathrm{d}$ \\
\hline $\mathrm{c}$ & $\{\mathrm{a}, \mathrm{c}, \mathrm{d}\}$ & $\{\mathrm{b}, \mathrm{d}\}$ & $\{\mathrm{a}, \mathrm{c}, \mathrm{d}\}$ & $\mathrm{d}$ \\
\hline $\mathrm{d}$ & $\{\mathrm{a}, \mathrm{c}, \mathrm{d}\}$ & $\mathrm{d}$ & $\{\mathrm{a}, \mathrm{c}, \mathrm{d}\}$ & $\mathrm{d}$
\end{tabular}

Table 1: Hyperoperation on a set

Then $(S, \star)$ is a semihypergroup.

Definition 2.5. Let a be an element in a semihypergroup $S$. We say that a is regular if and only if there exists $b \in S$ such that $a \in a \star b \star a$. $A$ semihypergroup $S$ is said to be regular if $\forall a \in S$ is regular.

The semihypergroup $S$ in Table 1 is regular where $a \in a \star a \star a, b \in b \star a \star b, c \in c \star d \star c$ and $d \in d \star c \star d$.

Definition 2.6. An idempotent in a semihypergroup $S$ is an element e such that $e \in e \star e$. $A$ nonempty set $A$ of $S$ is called idempotent subset if $A \subseteq A \star A$.

Definition 2.7. Let $S$ be a regular semihypergroup. If the set of idempotents $E$ of $S$ is a subsemihypergroup then we call $S$ an orthodox semihypergroup.

Definition 2.8. Let $x$ be an element of a semihypergroup $S$. We say that $x^{-1}$ is an inverse of $x$ if and only if $x \in x \star x^{-1} \star x$ and $x^{-1} \in x^{-1} \star x \star x^{-1}$.

Definition 2.9. A semihypergroup $S$ is said to be inverse if for each $x \in S$ there exists a unique inverse $x^{-1}$ in $S$.

Thus inverse semihypergroups are regular.

Example 2.10. Let $S=\{1,2,3\}$ be a semihypergroup defined by the following hyperoperation table:

\begin{tabular}{c|c|c|c}
$\star$ & 1 & 2 & 3 \\
\hline 1 & $\{2,3\}$ & 1 & 1 \\
\hline 2 & 1 & $\{2,3\}$ & 2 \\
\hline 3 & 1 & 2 & 3 \\
\hline
\end{tabular}

Table 2: Hyperoperation on a semihypergroup

Then $(S, \star)$ is an inverse semihypergroup as each element of $S$ has a unique inverse as it is shown in Table 2.

$$
1^{-1}=1, \quad 2^{-1}=2, \quad 3^{-1}=3 .
$$

Definition 2.11. Let $L$ be a nonempty set with a binary hyperoperation $\otimes$ on $L$ satisfying the following conditions, $\forall x, y, z \in L$,

- Idempotent; $x \in x \otimes x$.

- Commutative; $x \otimes y=y \otimes x$.

- Associative; $(x \otimes y) \otimes z=x \otimes$ $(y \otimes z)$.

Then $(L, \otimes)$ is called a hypersemilattice.

Example 2.12. Let $L=\{a, b, c, d\}$ be a set defined by the following hyperoperation table:

\begin{tabular}{c|c|c|c|c}
$\otimes$ & $\mathrm{a}$ & $\mathrm{b}$ & $\mathrm{c}$ & $\mathrm{d}$ \\
\hline $\mathrm{a}$ & $\mathrm{a}$ & $\mathrm{a}$ & $\mathrm{a}$ & $\mathrm{a}$ \\
\hline $\mathrm{b}$ & $\mathrm{a}$ & $\{\mathrm{a}, \mathrm{b}\}$ & $\mathrm{a}$ & $\{\mathrm{a}, \mathrm{b}\}$ \\
\hline $\mathrm{c}$ & $\mathrm{a}$ & $\mathrm{a}$ & $\mathrm{c}$ & $\mathrm{c}$ \\
\hline $\mathrm{d}$ & $\mathrm{a}$ & $\{\mathrm{a}, \mathrm{b}\}$ & $\mathrm{c}$ & $\{\mathrm{c}, \mathrm{d}\}$
\end{tabular}

Table 3: Hyperoperation on a set

Then $L$ with the operation $\otimes$ in Table 3 is a hypersemilattice. 


\section{Main Results}

Lemma 3.1. Let e be an idempotent of an orthodox semihypergroup $S$. Then, every inverse of $e$ is an idempotent.

Proof. Let $x$ be an inverse of $e$, then $e \in e \star$ $x \star e$ and $x \in x \star e \star x$. Thus

$$
e \star x \in e \star x \star e \star x
$$

and $x \star e \in x \star e \star x \star e$ and thus $e \star x$ and $x \star e$ are idempotents. Then we have

$$
\begin{aligned}
x & \in x \star e \star x \\
& \subseteq x \star e \star e \star x \\
& \subseteq E \star E \\
& \subseteq E
\end{aligned}
$$

Therefore $x$ is an idempotent.

Theorem 3.2. Let $S$ be an orthodox semihypergroup. Let $e, f \in E$ such that

$$
e \in e \star f \star e \text { and } f \in f \star e \star f .
$$

Then for any $a, b \in S$, any inverse $x$ of $a \star$ $e \star b$ is also an inverse of $a \star f \star b$.

Proof. By the assumption, we have

$$
x \in x \star(a \star e \star b) \star x
$$

and

$a \star e \star b \in(a \star e \star b) \star x \star(a \star e \star b)$.

Let $u$ and $v$ be the inverses of $a$ and $b$ respectively and thus

$$
a \in a \star u \star a \text { and } u \in u \star a \star u \text {. }
$$

And

$$
b \in b \star v \star b \text { and } v \in v \star b \star v .
$$

Therefore,

$$
\begin{aligned}
a \star e \star b \subseteq & (a \star e \star b) \star x \star(a \star e \star b) \\
\subseteq & a \star e \star(b \star v \star b) \star x \\
& \star(a \star u \star a) \star e \star b
\end{aligned}
$$

and thus

$$
\begin{aligned}
u \star a \star e \star b \star v \subseteq & \leq \star a \star e \star(b \star v \star b) \star x \\
& \star(a \star u \star a) \star e \star b \star v \\
= & (u \star a \star e \star b \star v) \star(b \\
& \star x \star a) \\
& \star(u \star a \star e \star b \star v) .
\end{aligned}
$$

And we have

$$
\begin{aligned}
b \star x \star a \subseteq & b \star(x \star a \star e \star b \star x) \star a \\
\subseteq & b \star x \star(a \star u \star a) \star e \\
& \star(b \star v \star b) \star x \star a \\
= & (b \star x \star a) \star(u \star a \star e \star b \star v) \\
& \star(b \star x \star a) .
\end{aligned}
$$

Which implies that $b \star x \star a$ and $u \star a \star e \star$ $b \star v$ are inverses. Since that $u \star a, e, b \star v \in$ $E$ then $u \star a \star e \star b \star v \in E$. And because that $u \star a \star e \star b \star v$ is the inverse of $b \star x \star a$ and it is idempotent then $b \star x \star a$ is an idempotent by Lemma 3.1, which means that $b \star x \star a$ is an inverse of $u \star a \star f \star b \star v$.

(Generally, $E \cong \sum E_{\lambda}: \lambda \in \Gamma, \Gamma$ is a hypersemilattice and each $E_{\lambda}$ is a rectangular band). Since $e \in e \star f \star e$ and $f \in f \star e \star f$, there exists $E_{\delta}$ such that $e, f \in E_{\delta}$. Thus for any $t \in E_{\alpha}$ and $g \in E_{\beta}$, we have $t \star e \star g$, $t \star f \star g \in E_{\alpha \delta \beta}$.

Therefore any idempotent $\tau$ which is an inverse of $\xi \star e \star \mu$ is also an inverse of $\xi \star f \star \mu$. where $\xi$ and $\mu$ are idempotents [18]. Hence we have

$$
\begin{aligned}
b \star x \star a \subseteq & (b \star x \star a) \\
& \star(u \star a \star f \star b \star v) \\
& \star(b \star x \star a)
\end{aligned}
$$$$
\begin{aligned}
u \star a \star f \star b \star v \subseteq & (u \star a \star f \star b \star v) \\
& \star(b \star x \star a) \\
& \star(u \star a \star f \star b \star v) .
\end{aligned}
$$

And 
also

$$
\begin{aligned}
a \star f \star b & \subseteq a \star f \star b \star x \star a \star f \star b \\
& \subseteq a \star f \star b \star x \star a \star f \star b \\
& \star x \star a \star f \star b
\end{aligned}
$$

and thus

$$
\begin{aligned}
x \star a \star f \star b \star x & \subseteq x \star a \star f \star b \\
& \star x \star a \star f \\
& \star b \star x \star a \\
& \star f \star b \star x
\end{aligned}
$$

which implies that $a \star f \star b$ and $x \star a \star$ $f \star b \star x$ are inverses. Similarly

$a \star e \star b \subseteq a \star e \star b \star x \star a$

$$
\star f \star b \star x \star a \star e \star b
$$

and then

$$
\begin{aligned}
x \star a \star e \star b \star x \subseteq & x \star a \star e \\
& \star b \star x \star a \\
& \star f \star b \star x \\
& \star a \star e \star b \star x .
\end{aligned}
$$

Since that $x$ is the inverse of $a \star e \star$ $b$ then $x$ is the inverse of $a \star f \star b$.

Proposition 3.3. Let $S$ be a semihypergroup. If $s \in S$ is an idempotent then $s^{-1}=s$.

Proof. Since $s$ is an idempotent then $s \in s \star$ $s$.Thus

$$
s \star s \subseteq s \star s \star s
$$

and then

$$
s \in s \star s \subseteq s \star s \star s .
$$

Therefore, $s^{-1}=s$.

Proposition 3.4. If $S$ is an inverse semihypergroup. Then $\forall s \in S: s \star s^{-1}$ is an idempotent.
Proof. Since $S$ is an inverse semihypergroup then

$$
s \in s \star s^{-1} \star s \text { and } s^{-1} \in s^{-1} \star s \star s^{-1}
$$

and then

$$
\begin{aligned}
s \star s^{-1} & \subseteq\left(s \star s^{-1} \star s\right) \star s^{-1} \\
& =\left(s \star s^{-1}\right) \star\left(s \star s^{-1}\right) . \\
\text { Let } x \in & s \star s^{-1} \text { which implies that } \\
x & \in\left(s \star s^{-1}\right) \star\left(s \star s^{-1}\right)
\end{aligned}
$$

and thus

$$
s \star s^{-1} \subseteq\left(s \star s^{-1}\right) \star\left(s \star s^{-1}\right) .
$$

Proposition 3.5. If $S$ is an inverse semihypergroup and $a, b$ are idempotents in $S$ then

$$
b \star(a \star b)^{-1} \star a
$$

is an idempotent inverse of $a \star b$.

Proof. Let $a, b$ be idempotents. Let $s$ be an inverse of $a \star b$ Which means $s=(a \star$ $b)^{-1}$. Thus,

$$
s \in s \star(a \star b) \star s,
$$

and

$$
a \star b \subseteq(a \star b) \star s \star(a \star b) .
$$

multiplying (1) by $b$ from the left and $a$ from the right, we have

$$
\begin{aligned}
b \star s \star a \subseteq & \subseteq \star(s \star a \star b) \star s \star a \\
\subseteq & b \star s \star(a \star a) \star(b \star b) \\
& \star s \star a \\
= & (b \star s \star a) \star(a \star b) \\
& \star(b \star s \star a)
\end{aligned}
$$


From (2) we have,

$$
\begin{aligned}
a \star b \subseteq & (a \star b) \star s \star(a \star b) \\
\subseteq & a \star(b \star b) \star s \\
& \star(a \star a) \star b \\
= & (a \star b) \star(b \star s \star a) \\
& \star(a \star b) .
\end{aligned}
$$

Therefore, $b \star(a \star b)^{-1} \star a$ is an idempotent inverse of $a \star b$.

Theorem 3.6. If $S$ is an inverse semihypergroup then its idempotents commute.

Proof. Let $a, b$ be idempotents. We prove that $a \star b=b \star a$. Hence

$$
(a \star b)^{-1}=b \star(a \star b)^{-1} \star a
$$

by Proposition 3.5 and uniqueness of inverses. Thus $(a \star b)^{-1}$ is idempotent and thus

$$
(a \star b)^{-1}=a \star b
$$

as every idempotent is it self inverse (Proposition 3.3) and the uniqueness. Similarly, $b \star a$ is an idempotent. Therefore,

$$
\begin{aligned}
b \star a & \subseteq b \star a \star b \star a \\
& \subseteq b \star a \star a \star b \star b \star a \\
& =(b \star a) \star(a \star b) \star(b \star
\end{aligned}
$$

Analogously,

$$
\begin{aligned}
a \star b & \subseteq a \star b \star a \star b \\
& \subseteq a \star b \star b \star a \star a \star b \\
& =(a \star b) \star(b \star a) \star(a \star b) .
\end{aligned}
$$

Thus $a \star b$ and $b \star a$ are inverses and then $a \star b=b \star a$.

Lemma 3.7. For any two elements $x, y$ in an inverse semihypergroup $S:(x \star y)^{-1}=y^{-1} \star$ $x^{-1}$.
Proof. Since

$x \in x \star x^{-1} \star x, y \in y \star y^{-1} \star y$

and

$x^{-1} \in x^{-1} \star x \star x^{-1}, y^{-1} \in y^{-1} \star y \star y^{-1}$.

Then we have

$$
\begin{aligned}
x \star y \subseteq & \left(x \star x^{-1} \star x\right) \star\left(y \star y^{-1} \star y\right) \\
= & x \star\left(x^{-1} \star x\right) \star\left(y \star y^{-1}\right) \star y \\
= & x \star\left(y \star y^{-1}\right) \star\left(x^{-1} \star x\right) \star y \\
& \quad(\text { since idempotents commute }) \\
= & (x \star y) \star\left(y^{-1} \star x^{-1}\right) \star(x \star y) .
\end{aligned}
$$

Similarly,

$$
\begin{aligned}
y^{-1} \star x^{-1} \subseteq & \left(y^{-1} \star y \star y^{-1}\right) \star\left(x^{-1} \star x \star x^{-1}\right) \\
= & y^{-1} \star\left(y \star y^{-1}\right) \star\left(x^{-1} \star x\right) \star x^{-1} \\
= & y^{-1} \star\left(x^{-1} \star x\right) \star\left(y \star y^{-1}\right) \star x^{-1} \\
& \quad(\text { since idempotents commute }) \\
= & \left(y^{-1} \star x^{-1}\right) \star(x \star y) \star\left(y^{-1} \star x^{-1}\right)
\end{aligned}
$$

Which implies that $(x \star y)^{-1}=y^{-1} \star x^{-1}$.

Proposition 3.8. All hypergroups are inverse semihypergroups and an inverse semihypergroup is a hypergroup if and only if it has a a). unique idempotent.

Proof. It is obvious that all hypergroups are inverse semihypergroups. Now we show the other direction. Let $S$ be an inverse semihypergroup with a unique idempotent $e$. Then by Proposition 3.4 we have,

$$
s \star s^{-1}=e=s^{-1} \star s .
$$

Then

$$
e \star s=\left(s \star s^{-1}\right) \star s .
$$

And as $s$ and $s^{-1}$ are inverses then

$$
s \in s \star s^{-1} \star s=e \star s,
$$


which implies that $s \in e \star s$ and similarly $s \in$ $s \star e$. Let $x \in e \star s$, then we have $x \in s \star s^{-1} \star$ $s$, and thus $e \star s \subseteq s \star e$ and similarly we can prove $s \star e \subseteq e \star s$. Therefore $e \star s=s \star e$.

Lemma 3.9. Let $S$ be an inverse semihypergroup then,

- For each idempotent $e$ and element $s$ there is an idempotent $f$ such that $e \star$ $s \subseteq s \star f$.

- For each idempotent $e$ and element $s$ there is an idempotent $f$ such that $s \star$ $e \subseteq f \star s$.

Proof. (1) We may put $f=s^{-1} \star e \star s$. Notice that $f$ is an idempotent because

$s \in s \star s^{-1} \star s$ and $s^{-1} \in s^{-1} \star s \star s^{-1}$. Then we have,

$$
\begin{aligned}
f= & s^{-1} \star e \star s \\
\subseteq & s^{-1} \star e \star s \star s^{-1} \star s \\
& \quad\left(\text { since } s \in s \star s^{-1} \star s\right) \\
\subseteq & s^{-1} \star e \star e \star s \star s^{-1} \star s \\
& \quad(\text { since e is an idempotent }) \\
\subseteq & \left(s^{-1} \star e \star s\right) \star\left(s^{-1} \star e \star s\right) \\
& \quad\left(\text { since e and } s \star s^{-1} \text { commute }\right) \\
= & f \star f .
\end{aligned}
$$

Therefore, $f$ is idempotent. Then

$$
\begin{aligned}
e \star s & \subseteq e \star\left(s \star s^{-1}\right) \star s \\
& =\left(s \star s^{-1}\right) \star e \star s \\
& \quad\left(\text { since e and } s \star s^{-1} \text { commute }\right) \\
& =s \star\left(s^{-1} \star e \star s\right) \\
& =s \star f .
\end{aligned}
$$

(2) is proved analogously.

Lemma 3.10. Let $S$ be an inverse semihypergroup and let $a, b \in S$ then;
- If there exists an idempotent $f$ such that $a=b \star f$ then $a \subseteq a \star a^{-1} \star b$.

- If there exists an idempotent e such that $a=e \star b$ then $a \subseteq b \star a^{-1} \star a$.

Proof. We show (1). Let $a=b \star f$ where $f$ is an idempotent. Then

$$
\begin{aligned}
a & =b \star f \\
& \subseteq\left(b \star b^{-1} \star b\right) \star f \\
& =b \star f \star\left(b^{-1} \star b\right)
\end{aligned}
$$

(since idempotents commute)

$$
\begin{aligned}
& \subseteq b \star f \star f \star b^{-1} \star b \\
& =b \star f \star f^{-1} \star b^{-1} \star b
\end{aligned}
$$

(since every idemotent is it self inverse)

$$
=(b \star f) \star(b \star f)^{-1} \star b
$$

(by Lemma 3.7)

$$
=a \star a^{-1} \star b .
$$

We show similarly (2). Let $a=e \star b$. Then

$$
\begin{aligned}
a= & e \star b \\
\subseteq & e \star\left(b \star b^{-1} \star b\right) \\
= & \left(b \star b^{-1}\right) \star e \star b \\
& (\text { since idempotents commute }) \\
\subseteq & \left(b \star b^{-1}\right) \star e \star e \star b \\
= & \left(b \star b^{-1}\right) \star e^{-1} \star e \star b
\end{aligned}
$$

(since every idemotent is it self inverse)

$$
=b \star(e \star b)^{-1} \star(e \star b)
$$

(by Lemma B.7)

$$
=b \star a^{-1} \star a \text {. }
$$

Definition 3.11. Let $(S, \circ)$ and $(T, \star)$ be two semihypersemigroups. A map $\phi: S \rightarrow$ $T$ is called a homomorphism if

$$
\forall x, y \in S: \phi(x \circ y) \subseteq \phi(x) \star \phi(y) .
$$


We call $\phi$ a strong homomorphism if

$\forall x, y \in S: \phi(x \circ y)=\phi(x) \star \phi(y)$.

We call $\phi$ isomorphism if $\phi$ is one-to-one onto strong homomorphism and denoted by $S \cong T$.

Example 3.12. Let $S=\{a, b, c\}$ and $T=$ $\{1,2,3\}$ be two semihypergroups with the following hyperoperation tables:

\begin{tabular}{c|c|c|c}
$\circ$ & $\mathrm{a}$ & $\mathrm{b}$ & $\mathrm{c}$ \\
\hline $\mathrm{a}$ & $\mathrm{a}$ & $\{\mathrm{a}, \mathrm{b}, \mathrm{c}\}$ & $\mathrm{c}$ \\
\hline $\mathrm{b}$ & $\{\mathrm{a}, \mathrm{b}, \mathrm{c}\}$ & $\mathrm{b}$ & $\{\mathrm{a}, \mathrm{b}, \mathrm{c}\}$ \\
\hline $\mathrm{c}$ & $\mathrm{c}$ & $\{\mathrm{a}, \mathrm{b}, \mathrm{c}\}$ & $\mathrm{c}$ \\
\hline
\end{tabular}

Table 4: Hyperoperation $\circ$ on $S$

\begin{tabular}{c|c|c|c}
$\star$ & 1 & 2 & 3 \\
\hline 1 & $\{1,3\}$ & $\{1,2,3\}$ & 3 \\
\hline 2 & $\{1,2,3\}$ & 2 & $\{1,2,3\}$ \\
\hline 3 & 3 & $\{1,2,3\}$ & 3 \\
\hline
\end{tabular}

Table 5: Hyperoperation $\star$ on $T$

Let $\phi: S \rightarrow T$ be a mapping defined by $\phi(a)=1, \phi(b)=2, \phi(c)=3$. Thus $\phi$ is a homomorphism but not a strong homomorphism (Table 4 and (Table 5).

Lemma 3.13. Let $\phi: S \rightarrow T$ be a homomorphism between two inverse semihypergroups $(S, \circ)$ and $(T, \star)$. Then

- $\phi\left(s^{-1}\right)=\phi(s)^{-1} \forall s \in S$.

- If $e$ is an idempotent then $\phi(e)$ is an idempotent.

- If $\phi$ is a strong homomorphism and $\phi(s)$ is an idempotent then there is an idempotent e such that $\phi(s) \subseteq \phi(e)$.

- Im $\phi$ is an inverse subsemihypergroup of $T$.
- If $U$ is an inverse subsemihypergroup of $T$ then $\phi^{-1}(U)$ is an inverse subsemihypergroup of $S$.

Proof. (1) Since $s \in s \circ s^{-1} \circ s$ and $s^{-1} \in$ $s^{-1} \circ s \circ s^{-1}$. Then

$$
\begin{aligned}
\phi(s) & \subseteq \phi\left(s \circ s^{-1} \circ s\right) \\
& \subseteq \phi(s) \star \phi\left(s^{-1}\right) \star \phi(s),
\end{aligned}
$$

and

$$
\begin{aligned}
\phi(s)^{-1} & \subseteq \phi\left(s^{-1} \circ s \circ s^{-1}\right) \\
& \subseteq \phi\left(s^{-1}\right) \star \phi(s) \star \phi\left(s^{-1}\right) .
\end{aligned}
$$

Thus $\phi(s)^{-1}=\phi\left(s^{-1}\right)$.

(2) Since $e \in e \circ e$ then $\phi(e) \subseteq \phi(e \circ$

e) $\subseteq \phi(e) \star \phi(e)$.

(3) Since $\phi(s)$ is an idempotent then

$$
\begin{aligned}
\phi(s) & \subseteq \phi(s) \star \phi(s) \\
& =\phi(s)^{-1} \star \phi(s) \quad \text { (from Proposition 3.3) } \\
& =\phi\left(s^{-1}\right) \star \phi(s) \\
& =\phi\left(s^{-1} \circ s\right)
\end{aligned}
$$

(4) Since $\phi$ is a semihypergroup homomorphism, $\operatorname{Im} \phi$ is a subsemihypergroup of $T$. By (1), $\operatorname{Im} \phi$ is closed under inverses.

(5) Obvious.

\section{4 conclusion}

Inverse semihypergroup is a new direction in the field of hyperstructures. The fine generalization of hypergroups is further organized by inverse semihypergroups. Although several hypergroup principle theorems have been effectively exported to inverse semihypergroup theory, there is little evidence of the contribution of hypersemillatice theory.

The study presented in this paper is an initial step in the theoretical investigation of inverse semihypergroups. We will be 
studying in the future the ability of describing inverse semihypergroup in term of their hypersemilattice of idempotents. Also an orthodox inverse semihypergroup is a rich new area of research. A simple question can be asked, can we generalize the theorems of band semigroups and rectangular band semigroups, to band semihypergroups and rectangular band semihypergroups?

\section{Acknowledgement}

This research was funded by the Deanship of Scientific Research at Princess Nourah bint Abdulrahman University through the Fast-track Research Funding Program.

\section{Funding}

This research was funded by the Deanship of Scientific Research at Princess Nourah bint Abdulrahman University through the Fast-track Research Funding Program.

\section{References:}

[1] F. Marty, Sur une generalization de la notion de groupe, 8th Congress Math. Scandinaves, Stockholm , 45-49, 1934.

[2] M. Benado. Asupra unei generălizari a noțiunii de structură, Bul. St., Sect. Mat. Fiz, 1953, v. 5, p. 41-48, 1953.

[3] M. Krasner. Approximation des corps valués complets de caractéristique $p \neq 0$ par ceux de caractéristique 0. In: Colloque d'Algebre Supérieure: tenu a Bruxelles du 19 au 22 décembre 1956. Bruxelles: Louvain (Belge): Ceuterick, v. 228, p. 129-206, 1957.

[4] M. Krasner. A class of hyperrings and hyperfields. Internat. J. Math. and Math. Sci., v. 6, n. 2, p. 307-312, 1983.

[5] B. Davvaz, Semihypergroup Theory, Academic Press, 2016.
[6] P. Corsini and V. Leoreanu, Applications of Hyperstructure Theory, Advances in Mathematics, vol 5, Kluwer Academic Publishers, Dordrecht, The Netherlands, 2003.

[7] P. Corsini, Sur les semi-hypergroupes, Atti della Società Peloritana di Scienze Fisiche, Matematiche e Naturali, vol. 26, pp. 363-372, 1980 (French).

[8] B. Davvaz and N. S. Poursalavati, Semihypergroups and Shypersystems, Pure Mathematics and Applications, vol. 11, pp.43-49, 2000.

[9] C. Gutan, Simplifable semihypergroups, Algebraic Hyperstructures and Applications (Xánthi, 1990), pp. 103-111, World Scientific Publishing, Teaneck, NJ, USA, 1991.

[10] V. Leoreanu, About the simplifable cyclic semihypergroups, Italian Journal of Pure and Applied Mathematics, no. 7, pp. 69-76, 2000.

[11] B. Davvaz, Some results on congruences on semihypergroups, Bulletin of the Malaysian Mathematical Sciences Society, Second Series, vol. 23, no. 1, pp. 53$58,2000$.

[12] P. Bonansinga and P. Corsini, On semihypergroup and hypergroup homomorphisms, Unione Matematica Italiana, Bollettino, B. Serie VI, vol. 1, no. 2, pp. 717-727, 1982 (Italian).

[13] D. Fasino and D. Freni, Existence of proper semihypergroups of type $U U$ on the right, Discrete Mathematics, vol. 307, no. 22, pp. 2826-2836, 2007.

[14] A. Hasankhani, Ideals in a semihypergroup and Green's relations, Ratio Mathematica, no. 13, pp. 29-36, 1999. 
[15] S. V. Onipchuk, Regular semihypergroups, Matematicheski Sbornik, vol. 183, no. 6, pp. 43-54, 1992 (Russian), translation in Russian Academy of Sciences. Sbornik Mathematics, vol. 76, pp. 155-164, 1993.

[16] S. Wannusit and P. Patthanangkoor, On Inverse Semipolygroups, Thai Journal of Science and Technology, 324-333, 2019.

[17] M.V. Lawson, Recent developments in inverse semigroup theory, Semigroup Forum 100, 103-118 (2020).

[18] M. Yamada, On a regular semigroup in which the idempotents form a band, $\mathrm{Pa}$ cific Journal of Mathematics, 33(1), 261$272,1970$.
Follow: www.wseas.org/multimedia/contributorrole-instruction.pdf

\section{Creative Commons Attribution License 4.0} (Attribution 4.0 International, CC BY 4.0)

This article is published under the terms of the Creative Commons Attribution License 4.0

https://creativecommons.org/licenses/by/4.0/deed.en_US 